\title{
Correction to: Transcriptional suppression of androgen receptor by 18ß-glycyrrhetinic acid in LNCaP human prostate cancer cells
}

\author{
You $\mathrm{Sun}^{3} \cdot$ Pramod Aryal ${ }^{6,8} \cdot$ Mingyue Jiang ${ }^{3}$. \\ Shuang $\mathrm{Wu}^{3,7} \cdot$ Pil-Hoon Park ${ }^{5}$ Kyung Song ${ }^{1,2,3,4,6}$
}

Published online: 31 July 2020

(c) The Pharmaceutical Society of Korea 2020
The authors regret to inform that authors Pramod Aryal and Shuang $\mathrm{Wu}$ were inadvertently omitted from authorship of the manuscript above at the time of original submission. Pramod Aryal and Shuang Wu were contributors to conducting experiments, acquiring and initial plotting/ analyzing data. The authors would like to state that Sun You and Pramod Aryal contributed equally to this work as co-first authors. The authors would like to re-designate the authorship of this manuscript, and add the current addresses of these two new authors, Pramod Aryal and Shuang Wu as shown in this Correction.

The authors would like to sincerely apologize for any inconvenience and confusion this may have caused.

Publisher's Note Springer Nature remains neutral with regard to jurisdictional claims in published maps and institutional affiliations.

The authors You Sun and Pramod Aryal have contributed equally to this work.

The original article can be found online at https://doi. org/10.1007/s12272-020-01228-z.

Kyung Song

kyungsong@wku.ac.kr

1 Department of Pharmacy, College of Pharmacy, Wonkwang University, Jeollabuk-do, Iksan 54538, Republic of Korea

2 Institute of Pharmaceutical Research and Development, Wonkwang University, Jeollabuk-do, Iksan 54538, Republic of Korea

3 Department of Herbal Resources, Professional Graduate School of Oriental Medicine, Wonkwang University, Jeollabuk-do, Iksan 54538, Republic of Korea

4 Integrated Omics Institute, Wonkwang University, Jeollabuk-do, Iksan 54538, Republic of Korea
Department of Pharmacy, Yeungnam University, Gyeongsan, Gyeongbuk 38541, Republic of Korea

6 Department of Pharmacy, Graduate School of Wonkwang University, Jeollabuk-do, Iksan 54538, Republic of Korea

7 Present Address: Division of Jilin Province, Hunchun Natural Resources, Hunchun, Jilin Province 133300, People's Republic of China

8 Present Address: Department of Biochemistry and Molecular Biology, Monash University, Clayton, VIC 3800, Australia 